CERN-EP/2002-049

3 July 2002

\title{
ON-LINE YIELDS OBTAINED WITH THE ISOLDE RILIS
}

\author{
U. Köster ${ }^{1)}$ V.N. Fedoseyev ${ }^{1) 2}$ A.N. Andreyev ${ }^{3)}$ U.C. Bergmann ${ }^{1)}$ R. Catherall ${ }^{1)}$
} J. Cederkäll ${ }^{1)}$ M. Dietrich ${ }^{1)}$ H. De Witte ${ }^{4)}$ D.V. Fedorov ${ }^{5}$ L. Fraile ${ }^{1)}$ S. Franchoo $^{1)}$ H. Fynbo ${ }^{1)}$ U. Georg ${ }^{1)}$ T. Giles ${ }^{1)}$ M. Gorska ${ }^{4)}$ M. Hannawald ${ }^{6}$ M. Huyse $^{1) 4}$ A. Joinet ${ }^{1)}$ O.C. Jonsson ${ }^{1)}$ K.L. Kratz ${ }^{6)}$ K. Kruglov ${ }^{4)}$ Ch. Lau') J. Lettry ${ }^{1)}$ V.I. Mishin ${ }^{2)}$ M. Oinonen ${ }^{1)}$ K. Partes ${ }^{1)}$ K. Peräjärvi ${ }^{1)}$ B. Pfeiffer ${ }^{6)}$ H.L. Ravn ${ }^{1)}$ M.D. Seliverstov ${ }^{5)}$

P. Thirolf ${ }^{7)}$ K. Van de Vel ${ }^{4)}$ P. Van Duppen ${ }^{4}$ J. Van Roosbroeck ${ }^{4}$ L. Weissman ${ }^{1)}$ for the IS365, IS387, IS393 and ISOLDE Collaborations ${ }^{1)}$

\begin{abstract}
The ISOLDE resonance ionization laser ion source (RILIS) allows to ionize efficiently and selectively many metallic elements. In recent yield surveys and online experiments with the ISOLDE RILIS we observed ${ }^{23-34} \mathrm{Mg},{ }^{26-34} \mathrm{Al},{ }^{98-132} \mathrm{Cd}$, ${ }^{149} \mathrm{~Tb},{ }^{155-177} \mathrm{Yb},{ }^{179-200} \mathrm{Tl},{ }^{183-215} \mathrm{~Pb}$ and ${ }^{188-218} \mathrm{Bi}$. The obtained yields are presented together with measured release parameters which allow to extrapolate the release efficiency towards more exotic (short-lived) nuclides of the same elements.
\end{abstract}

(Submitted to Nuclear Instruments and Methods in Physics Research B) 


\section{Introduction}

The resonance ionization laser ion source (RILIS) [1] provides an efficient, fast and selective way to ionize many metallic elements. The elements ionized with the ISOLDE RILIS were reviewed recently $[2,3,4]$. In this article we will discuss on-line results, i.e. release curves and yields measured in recent target tests, nuclear spectroscopy experiments and on-line collections.

The measured release curves were fitted with the empiric 4-parameter-formula described in $[5,6]$. Table 1 shows a summary of the release parameters: $t_{r}$ the rise time, $t_{f}$ the fall time of the fast component, $t_{s}$ the fall time of the slow component and $\alpha$ the relative intensity of the fast component. Parameters which had to be fixed to obtain a stable fit are marked in parentheses.

The release parameters were used to determine for each radio-isotope the fraction which is released before it decays. The on-line efficiency of the RILIS cannot be easily monitored as in the case of a plasma ion source where the support gas is injected at a well-defined flowrate. Still a rough estimate can be made by scaling the off-line measured efficiency with the laser power available in the on-line run. Also the ion yield of a longlived isotope with known production cross-section gives an indication of the ionization efficiency. Correcting the ion yields for the released fraction and the ionization efficiency allows to deduce the in-target production yield. In some of the following graphs both values are plotted: the ion yield, showing the presently available radioactive ion beam intensity for the user, and the in-target production yield showing the theoretical limit that could be obtained with an ideal target (no decay losses) of the same thickness and dimensions coupled to an ideal ion source (100\% efficiency). The ratio between both curves shows the overall efficiency of the present target and ion source unit.

\section{$2 \quad$ Magnesium}

Neutron-rich Mg beams have been produced formerly at ISOLDE SC [7] from a $\mathrm{UC}_{x}$ /graphite target with a chemically unselective "hot plasma" ion source (MK5) [8]. Although this ion source is optimized for the production of singly charged ions, it also produces with less efficiency multiply charged ions. Hence, at a mass separator setting $A / q=31$ in addition to most $A=31$ isobars also doubly charged ions of mass 62 (e.g. ${ }^{62} \mathrm{Mn}^{2+}$ ), triply charged ions of mass 93 (e.g. ${ }^{93} \mathrm{Kr}^{3+}$ ), quadruply charged ions of mass 124 (e.g. ${ }^{124} \mathrm{Sb}^{4+}$ ) and molecules (e.g. ${ }^{12} \mathrm{C}^{19} \mathrm{O}^{+}$) may appear. In particular the contamination from abundantly produced fission products may be disturbing. Special ion source tuning can reduce this kind of background, but it cannot suppress it completely.

With the RILIS there is much less background of multiply charged ions. The mainly occuring beam contaminants are the surface ionized $\mathrm{Na}$ and $\mathrm{Al}$ isobars, the intensity of the former being about one order of magnitude lower compared to $\mathrm{Mg}$. The measured $\mathrm{Mg}$ ion yields and the deduced in-target production rates are shown in Fig. 1.

The pure RILIS Mg beams were used for precision mass measurements with the RF spectrometer MISTRAL [9]. In a former beamtime with a hot plasma ion source ${ }^{32} \mathrm{Mg}$

1) ISOLDE, CERN, 1211 Genève 23, Switzerland

2) Institute of Spectroscopy, Russian Academy of Sciences, 142190 Troitsk, Russia

3) Oliver Lodge Laboratory, University of Liverpool, Liverpool, L69 3BK, UK

4) Instituut voor Kern- en Stralingsfysica, K.U. Leuven, 3001 Leuven, Belgium

5) Petersburg Nuclear Physics Institute, 188350 Gatchina, Russia

6) Institut für Kernchemie, Universität Mainz, 55128 Mainz, Germany

7) Sektion Physik, Ludwig-Maximilians-Universität München, 85748 Garching, Bavaria 
could already be measured, but the strong background had to be reduced with narrow slit settings which compromised the transmission and hence the statistics. With the RILIS beams the slits could be opened wider and the statistics was significantly improved. Thus also the mass of the $N=21$ isotope ${ }^{33} \mathrm{Mg}$ could be measured for the first time [10].

\section{$3 \quad$ Aluminum}

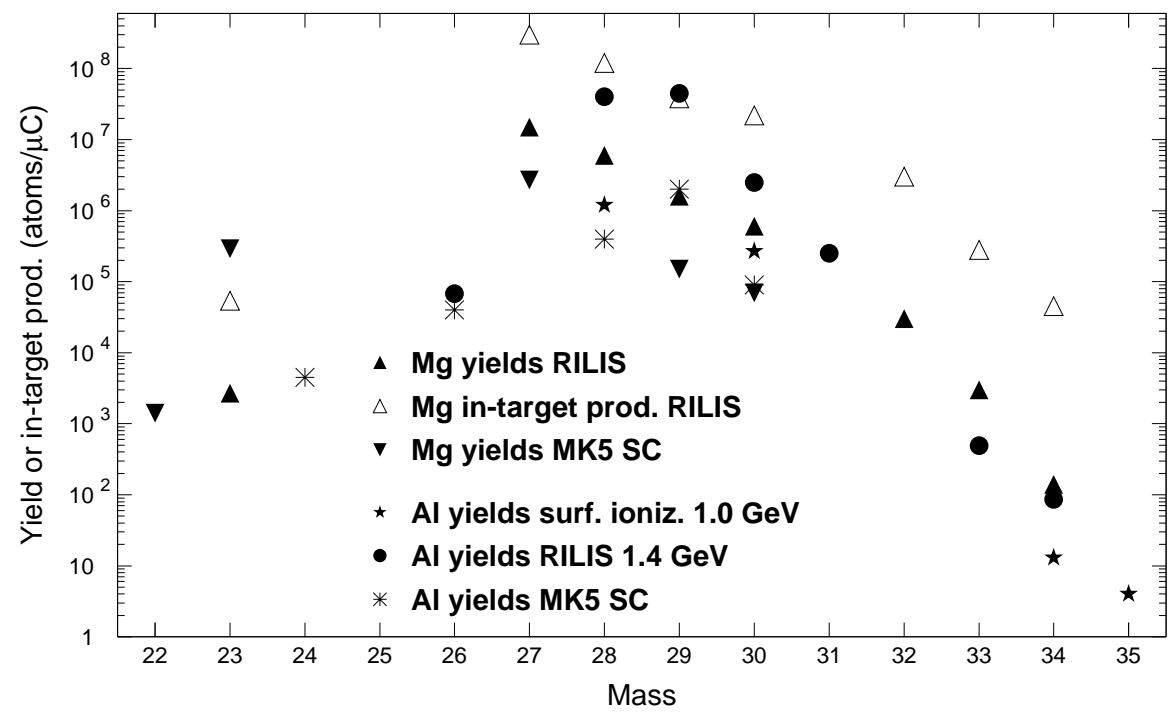

Figure 1: Ion yields and in-target production rates of magnesium and aluminum isotopes. For ${ }^{26} \mathrm{Al}$ only the yield of the $6.3 \mathrm{~s}$ isomeric state was measured.

$\mathrm{Al}$ is moderately well surface ionized. Neutron-rich $\mathrm{Al}$ beams up to ${ }^{35} \mathrm{Al}[11]$ were already studied at ISOLDE from a $\mathrm{UC}_{x}$ /graphite target with $\mathrm{W}$ surface ionizer. These yields obtained with a $1.0 \mathrm{GeV}$ proton beam are shown in Fig. 1 compared to yields from a MK5 ion source at ISOLDE SC.

Off-line tests had shown that the RILIS can increase the $\mathrm{Al}$ ionization efficiency by up to a factor 70 compared to surface ionization in a $2200{ }^{\circ} \mathrm{C}$ hot $\mathrm{W}$ cavity [3]. This was obtained by simultaneous excitation of the two fine structure levels of the ground state with two frequency doubled dye laser beams (308.2 and $309.3 \mathrm{~nm}$ respectively). For the on-line tests (dedicated for another purpose) only the $308.2 \mathrm{~nm}$ beam was used to excite the less populated fine structure level. Moreover the second step laser power focused to the HRS frontend was lower, giving a "laser on" "laser off" ratio of only about three. There is no doubt that the shown yields will be tripled when using both laser beams simultaneously. Even more could be gained by increasing the power focused to the target. Fig. 1 shows the yields measured with the $308.2 \mathrm{~nm}$ beam only.

Due to long desorption times, elemental $\mathrm{Al}$ is released very slowly ${ }^{1)}$. Adding $\mathrm{CF}_{4}$ will considerably speed up the release of $\mathrm{Al}$, then as AlF molecule [12]. To allow for ionization with the RILIS (tuned to excite atomic $\mathrm{Al}$ ) the AlF molecule needs to be dissociated in the ionizer cavity. A successful combination of fluorination and RILIS would increase the beam intensity of short-lived $\mathrm{Al}$ isotopes enormously and would allow to produce beams well beyond ${ }^{36} \mathrm{Al}$.

1) With the available tracer isotopes no indication for a fast release component was observed, hence $\alpha=0$ was assumed for the fit. 
Neutron-deficient $\mathrm{Cd}$ nuclides were one of the first beams separated at ISOLDE [13]. Concerning beam intensity and purity, these beams from a molten tin target are still showpieces at ISOLDE. A drawback of the molten targets is their rather slow release time of the order of tens of seconds to minutes [14]. Thus, considerable decay losses occur for the isotopes below ${ }^{102} \mathrm{Cd}$.

A $\mathrm{LaC}_{x}$ /graphite target (molar ratio La:C $\approx 1: 4$ ) combined with the RILIS was tested $^{2)}$. Due to a reduced target density $\left(\approx 0.22 \mathrm{~mol} / \mathrm{cm}^{2}\right.$ La versus $\left.1 \mathrm{~mol} / \mathrm{cm}^{2} \mathrm{Sn}\right)$ and smaller cross-sections the yields of ${ }^{100+x} \mathrm{Cd}$ are lower than from molten Sn targets, see Fig. 2. For ${ }^{98,99} \mathrm{Cd}$ they are comparable, but the most short-lived isotope ${ }^{97} \mathrm{Cd}$, where the faster release should give the biggest advantage, was not observed in this test [15]. With an efficient and selective identification method (e.g. detection of $\beta \mathrm{p}$ ) the RILIS could be used to perform atomic spectroscopy directly in-source [4], i.e. to measure magnetic moments and nuclear charge radii of the very neutron-deficient $\mathrm{Cd}$ isotopes ${ }^{97+x} \mathrm{Cd}$ around the $N=50$ shell closure.

Neutron-rich $\mathrm{Cd}$ isotopes up to ${ }^{130} \mathrm{Cd}$ had already been separated at the ISOLDE $\mathrm{SC}$ with a $\mathrm{UC}_{x} /$ graphite cloth target and a plasma ion source [16]. Some chemical selectivity was provided by a quartz transfer line at $600{ }^{\circ} \mathrm{C}$, suppressing part of the indium and cesium isobars but leaving (on mass 130) still a molecular beam of ${ }^{40} \mathrm{Ca}^{90} \mathrm{Br}^{+}$as background. To detect the heavier $\mathrm{Cd}$ isotopes with still shorter lifetimes the decay losses have to be minimized by keeping the full target and ion source unit at a sufficiently high temperature. Using the chemical selectivity of the RILIS, the short-lived neutron activities of the isotopes ${ }^{130-132} \mathrm{Cd}$ could thus be identified [17].

Fig. 2 shows the yields of Cd isotopes obtained with the RILIS at ISOLDE PSB. These yields were measured with four different targets, fluctuating ${ }^{3)}$ by up to an order of magnitude, probably due to a varying on-line ionization efficiency. Using a "neutron converter" [18], the yields are lower than in direct bombardment, but the isobaric background of neutron-deficient Cs is suppressed considerably.

The RILIS provides now beams free of molecular contaminations but leaves most of the In and Cs isobars in the beam. The ultimate beam purity could be obtained by combining a chemically selective transfer line (suppressing Cs and In) with the hot cavity RILIS (suppressing most molecules).

\section{$5 \quad$ Terbium}

Neutron-deficient $\mathrm{Tb}$ isotopes are abundantly produced by spallation of a Ta target and moderately well surface-ionized. The $4.1 \mathrm{~h}$ isomer $\left(1 / 2^{+}\right)$of ${ }^{149} \mathrm{~Tb}$ has a $17 \%$ branching ratio for alpha decay [19] and is therefore considered for radiotherapeutic applications [20]. A recent in vivo experiment at the University Hospital of Geneva using ${ }^{149} \mathrm{~Tb}$ marked antibodies gave very promising results for the treatment of lymphoma [21]. To obtain sufficient quantities of ${ }^{149} \mathrm{~Tb}$ for therapeutic purposes any possible increase in yield is important. Therefore we tested on-line the prospects of the RILIS for Tb ionization. We used an ionization scheme which had been developed in Troitsk [22]: three visible transitions $(579.6 \mathrm{~nm}, 551.7 \mathrm{~nm}$ and $618.3 \mathrm{~nm}$ ) leading to an autoionizing state. Due to the presence of many surface ionized isobars and oxides in the lanthanide region

2) This run was performed with $1.0 \mathrm{GeV}$ protons, all other yields shown in this paper were measured with $1.4 \mathrm{GeV}$ protons.

3) Also the fitted release times of Cd fluctuate by up to a factor two when comparing the different targets at different "age". Table 1 shows an average. 


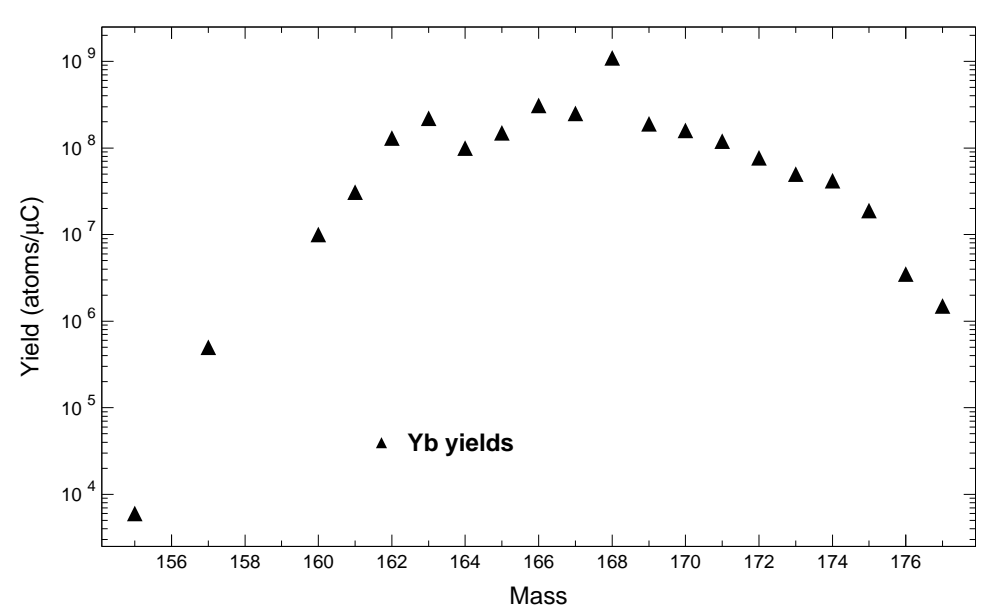

Figure 3: Ion yields of ytterbium isotopes from a Ta foil target.

\section{Thallium}

$\mathrm{Tl}$ is moderately well surface ionized and clean $\mathrm{Tl}$ beams were already available at ISOLDE SC. Off-line it was shown that the RILIS allows to increase the Tl ionization efficiency by about a factor 20 compared to surface ionization in a $2200{ }^{\circ} \mathrm{C}$ hot $\mathrm{W}$ cavity. In the on-line experiment the laser power was lower, but the surface ionization efficiency in the $2100{ }^{\circ} \mathrm{C}$ hot $\mathrm{Nb}$ cavity was also reduced, providing again a selectivity of about 20 .

Fig. 4 shows the measured yields of neutron-deficient $\mathrm{Tl}$ isotopes from a $\mathrm{W}$ surface ionizer and the RILIS respectively. In cases of several detected isomers the sum yield is shown. The yields of ${ }^{179-181} \mathrm{Tl}$ were estimated from the observed alpha emission rate and assumed branching ratios for $\alpha$ decay of $80 \%\left({ }^{179} \mathrm{Tl}\right), 10 \%\left({ }^{180} \mathrm{Tl}\right)$ and $20 \%\left({ }^{181 g} \mathrm{Tl}\right)$. For comparison are shown the yields of surface ionized $\mathrm{Tl}$ isotopes which were obtained at the IRIS facility by $1.0 \mathrm{GeV}$ proton induced spallation of a special $10 \mathrm{~g} / \mathrm{cm}^{2} \mathrm{ThC}_{x}$ target (produced by pyrolysis of a thorium diphtalocyanine precursor) coupled to a $\mathrm{W} /$ Re surface ionizer $[24,25]$.

\section{Lead and Bismuth}

$\mathrm{Pb}$ and $\mathrm{Bi}$ beams, ionized with an unselective hot plasma ion source, were available before at ISOLDE [7]. However, the admixture of many isobars ( $\mathrm{Hg}, \mathrm{Tl}, \mathrm{Po}$, At, etc.) made them difficult to use for nuclear spectroscopy and other applications. The use of the RILIS allows now to provide rather pure beams of $\mathrm{Pb}$ and $\mathrm{Bi}$, the remaining isobaric contaminations being the surface ionized $\mathrm{Tl}$ and Fr. The former is mostly on an acceptable level, but the latter is intense enough to be disturbing for most applications at $A>206$. Only for masses 215 to 218 the Fr isotopes are so short-lived that this background can be suppressed completely by a delayed opening of the electrostatic beamgate [26].

In cases of several detected ${ }^{7)}$ isomers the sum yield is shown, see Fig. 4. In most measured cases the yield of the high-spin isomer was higher. The yields of ${ }^{188-192} \mathrm{Bi}$ were determined from the alpha decay rates, assuming $\approx 100 \%$ branching ratios for $\alpha$ decay of ${ }_{188 g, 188 m, 189 g, 189 m} \mathrm{Bi}$. Note that the laser power in the Bi run was lower than in the former off-line experiment.

The RILIS can also be used to produce isomerically pure beams by tuning the

7) Not always could all isomers be detected. This causes the fluctuations between neighboring isotopes, as apparent in the plotted yield curve. 
wavelength of the resonantly exciting laser to the hyperfine structure of one or another isomer [27]. For the heavier elements $\mathrm{Tl}, \mathrm{Pb}$ and $\mathrm{Bi}$ the Doppler broadening is rather small compared to the hyperfine splitting and this technique works particularly well. It was recently used to perform nuclear and atomic spectroscopy on two isomers in ${ }^{185} \mathrm{~Pb}$ $[28]$.

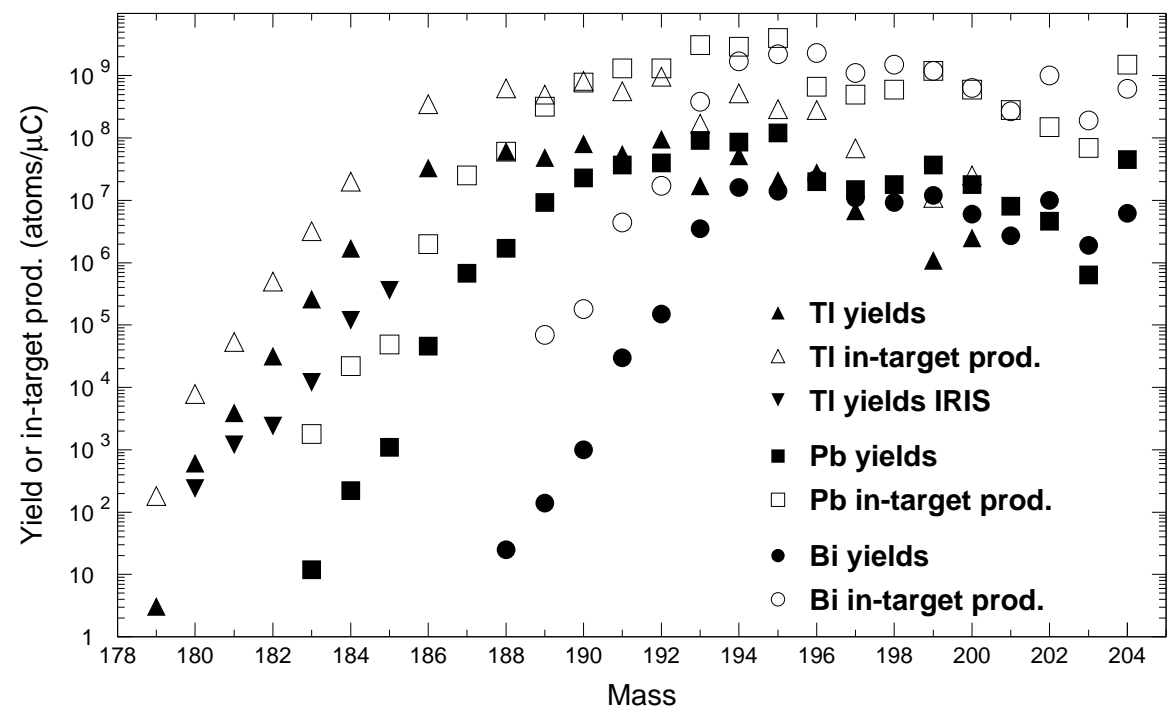

Figure 4: Ion yields and in-target production rates of neutron-deficient thallium, lead and bismuth isotopes. The yields of ${ }^{215} \mathrm{~Pb},{ }^{215 m} \mathrm{Bi}$ and ${ }^{218} \mathrm{Bi}$ (not plotted) were 0.3, 1400 and 44 per $\mu \mathrm{C}$ respectively.

\section{$9 \quad$ Summary}

This article summarizes release functions of eight different elements and the yields of over hundred isotopes which were measured with the ISOLDE RILIS in the last years. Detailed data of the individual experiments are shown in table 1.

Table 1: Overview of target characteristics, operation conditions, release parameters and estimated ionization efficiencies during the different on-line runs. Note the uncertainty in the determination of the ionization efficiency: about a factor two. The $\mathrm{UC}_{x} /$ graphite targets contain about $10 \mathrm{~g} / \mathrm{cm}^{2}$ of carbon in addition to the $\approx 50 \mathrm{~g} / \mathrm{cm}^{2}{ }^{238} \mathrm{U}$.

\begin{tabular}{|l|c|c|c|c|c|c|c|c|c|c|c|}
\hline $\begin{array}{l}\text { Ele- } \\
\text { ment }\end{array}$ & $\begin{array}{l}\text { Ion. } \\
\text { pot. } \\
\end{array}$ & \multicolumn{3}{|c|}{ Target } & \multicolumn{2}{c|}{ Ionizer } & \multicolumn{1}{c|}{$\begin{array}{c}\text { Ion. } \\
\text { eff. }\end{array}$} & $\alpha$ & \multicolumn{4}{|c|}{$\begin{array}{c}t_{r} \\
t_{f}\end{array}$} & $\begin{array}{c}t_{s} \\
\mathrm{~ms}\end{array}$ & $\mathrm{~ms}$ \\
\hline $\mathrm{Mg}$ & 7.65 & $\mathrm{UC}_{x} /$ gr. & 50 & 2020 & $\mathrm{~W}$ & 2200 & 10 & 0.98 & 66 & 190 & 860 \\
$\mathrm{Al}$ & 5.99 & $\mathrm{UC}_{x} / \mathrm{gr}$. & 49 & 2070 & $\mathrm{~W}$ & 2250 & $1-10$ & $(0)$ & $(100)$ & - & 22000 \\
$\mathrm{Cd}$ & 8.99 & $\mathrm{UC}_{x} /$ gr. & 50 & $\approx 2000$ & $\mathrm{Nb}$ & $\approx 2000$ & 10 & 0.98 & $\approx 60$ & $\approx 300$ & $\approx 3000$ \\
$\mathrm{~Tb}$ & 5.86 & $\mathrm{Ta}$ foil & 42 & 2200 & $\mathrm{~W}$ & 1900 & $?$ & - & - & - & - \\
$\mathrm{Yb}$ & 6.25 & $\mathrm{Ta}$ foil & 42 & 2150 & $\mathrm{~W}$ & 2000 & $\leq 15$ & 0.91 & $(300)$ & 100 & 35000 \\
$\mathrm{Tl}$ & 6.11 & $\mathrm{UC}_{x} / \mathrm{gr}$. & 50 & 2000 & $\mathrm{Nb}$ & 2100 & 5 & 0.98 & 45 & 600 & 5700 \\
$\mathrm{~Pb}$ & 7.42 & $\mathrm{UC}_{x} / \mathrm{gr}$. & 50 & 2050 & $\mathrm{Nb}$ & 2100 & 3 & 0.96 & 70 & 580 & 4700 \\
$\mathrm{Bi}$ & 7.29 & $\mathrm{UC}_{x} /$ gr. & 50 & 2050 & $\mathrm{Nb}$ & 2100 & 1 & 0.86 & 45 & 540 & 8000 \\
\hline
\end{tabular}




\section{Acknowledgements}

These yield measurements profited from support by the EU-RTD projects EURISOL (HPRI-CT-1999-50001) and TARGISOL (HPRI-CT-2001-50033) and by the BMBF under grant $05 \mathrm{KK} 1 \mathrm{TSA} / 7$.

\section{References}

[1] V.I. Mishin et al., Nucl. Instr. Meth. B 73 (1993) 550-560.

[2] V.N. Fedoseyev et al., Hyp. Int. 127 (2000) 409-416.

[3] U. Köster, Nucl. Phys. A 701 (2002) 441-451.

[4] V.N. Fedoseyev et al., Nucl. Instr. Meth. B, these proceedings.

[5] J. Lettry et al., Nucl. Instr. Meth. B 126 (1997) 130-134.

[6] U.C. Bergmann, Nucl. Instr. Meth. B, these proceedings.

[7] E. Hagebø et al., Nucl. Instr. Meth. B 70 (1992) 165-174.

[8] S. Sundell et al., Nucl. Instr. Meth. B 70 (1992) 160-164.

[9] D. Lunney et al., Phys. Rev. C 64 (2001) 054311.

[10] D. Lunney, CSNSM Orsay, priv. comm.

[11] S. Nummela et al., Phys. Rev. C 63 (2001) 044316.

[12] R. Kirchner, Nucl. Instr. Meth. B 126 (1997) 135-140.

[13] P.G. Hansen et al., Phys. Lett. 28B (1969) 415-419.

[14] J. Lettry et al., Nucl. Instr. Meth. B 126 (1997) 170-175.

[15] U. Köster, Ph.D. thesis, TU München (2000).

[16] K.L. Kratz et al., Z. Phys. A 325 (1986) 489-490.

[17] M. Hannawald et al., Phys. Rev. C 62 (2000) 054301.

[18] R. Catherall et al., Nucl. Instr. Meth. B, these proceedings.

[19] G. Audi et al., Nucl. Phys. A 624 (1997) 1-124.

[20] G.J. Beyer, Hyp. Int. 129 (2000) 529-553.

[21] G.J. Beyer et al., to be published.

[22] V.N. Fedoseyev et al., J. Phys. B 24 (1991) 1575-1583.

[23] A.E. Barzakh et al., Proc. of ENAM-01 (2002) in press.

[24] V.A. Bolshakov et al., Nucl. Instr. Meth. B 70 (1992) 69-74.

[25] A.E. Barzakh et al., Nucl. Instr. Meth. B 126 (1997) 150-153.

[26] P. Van Duppen et al., Nucl. Instr. Meth. B 134 (1998) 267-270.

[27] U. Köster et al., Nucl. Instr. Meth. B 160 (2000) 528-535.

[28] A.N. Andreyev et al., Eur. Phys. J. A 14 (2002) 63-75. 